\title{
Intraoperative optical coherence tomography to evaluate the effect of the eyelid speculum on corneal pachymetry during accelerated corneal cross-linking $\left(9 \mathrm{~mW} / \mathrm{cm}^{2}\right)$
}

R Ghaffari ${ }^{1}$, M Mortazavi ${ }^{1}$, P Anvari ${ }^{1}$, A Salamat Rad ${ }^{1}$, F Alipour ${ }^{1}$, F Hafezi ${ }^{2,3,4,5}$, S Asgari ${ }^{6}$ and $\mathrm{H}$ Hashemi ${ }^{6}$

\begin{abstract}
Purpose To investigate intraoperative anterior segment optical coherence tomography (AS-OCT) to compare pachymetry changes between two methods (group 1: speculum-on, group 2: speculum-off during riboflavin instillation stage) during corneal cross-linking /CXL) $\left(9 \mathrm{~mW} / \mathrm{cm}^{2}\right.$ for $10 \mathrm{~min}$ ) in keratoconic patients.

Methods In this interventional case series, 11 eyes (11 patients) in group 1 and 14 eyes (14 patients) in group 2 were enrolled. Pachymetry measurements by spectral domain OCT was performed at baseline, after epithelial removal, after $30 \mathrm{~min}$ of imbibition with riboflavin, and after UV irradiation.

Repeated measures analysis of variance was used to compare the trend of changes in different stages of the procedure within and between two groups.

Results The corneal thinnest pachymerty (CTP) at baseline, after epithelial removal, $30 \mathrm{~min}$ of riboflavin instillation, and irradiation was $469.8 \pm 26.5,438.0 \pm 27.1$, $354.3 \pm 28.4,367.1 \pm 31.8$ microns in group 1 and $463.2 \pm 25.1,438.0 \pm 27.1,421.6 \pm 54.0$, $386.9 \pm 34.0$ microns in group 2 , respectively. Group 1 showed a significantly greater decrease in the corneal pachymetry $(P<0.001)$ during riboflavin instillation, while in group 2 a greater decrease in CTP was observed during the UV irradiation phase $(P=0.002)$.

Conclusions Using the intraoperative OCT, the speculum-off group had a more stable corneal pachymetry during riboflavin
\end{abstract}

instillation time. Despite this, the effect was not lasting throughout the procedure; significant changes were noted in the corneal pachymetry at the end of operation in both groups; further highlighting the limitations imposed by corneal stromal shrinkage using isotonic dextran containing riboflavin solution for CXL.

Eye (2018) 32, 579-585; doi:10.1038/eye.2017.243;

published online 24 November 2017

\section{Introduction}

Keratoconus is a bilateral, progressive, noninflammatory, ectatic disease characterized by progressive corneal thinning and irregular astigmatism, due to biomechanical instability of the cornea. ${ }^{1}$ Biomechanical reinforcement of the cornea by collagen cross-linking (CXL) with riboflavin and ultraviolet $\mathrm{A}$ (UV-A) irradiation ${ }^{2,3}$ is a well-established treatment, and long-term studies have demonstrated its safety and efficacy at halting the progression of keratoconus, ${ }^{4,5}$ provided that relevant and detailed safety criteria are taken into account.

Corneal pachymetry is one of the most important factors with respect to the safety of CXL; a minimum thickness of $400 \mu \mathrm{m}$ is recommended to ensure the safety of the procedure and avoid the potential toxic effects of UV-A irradiation. ${ }^{3,6}$ In addition to the initial pachymetry, a significant change in corneal pachymetry may be induced during the different steps of the procedure. ${ }^{7-9}$ These changes include epithelial removal, dehydration due to corneal
${ }^{1}$ Eye Research Center, Farabi Eye Hospital, Tehran University of Medical Sciences, Tehran, Iran

${ }^{2}$ Laboratory for Ocular Cell Biology Laboratory, University of Geneva, Geneva, Switzerland

${ }^{3}$ Center for Applied Biotechnology and Molecular Medicine, University of Zurich, Zurich, Switzerland

${ }^{4}$ Department of Ophthalmology, University of Southern California Los Angeles, CA, USA

${ }^{5}$ ELZA Institute, Dietikon, Switzerland

${ }^{6}$ Noor Ophthalmology Research Center, Noor Eye Hospital, Tehran, Iran

Correspondence:

H Hashemi, Noor Ophthalmology Research Center, Noor Eye Hospital, No. 96 Esfandiar Boulevard, Vali'asr Avenue, Tehran, Iran

Tel: +98 2188651515 ; Fax: +98 2188651514 E-mail: hhahsemi@norc.ac.ir

Received: 4 July 2016 Accepted in revised form: 8 October 2017

Published online: 24 November 2017 
exposure, osmotic effects of the riboflavin-dextran solution, and UV irradiation, which all could potentially jeopardize the safety of the procedure, highlighting the important role of intraoperative pachymetric monitoring during the operation.

In this respect, previous studies point have noted the significant role played by corneal exposure during the imbibition period, which is one factor that induces corneal shrinkage within the presence of the standard isotonic riboflavin-dextran solution and epithelium-off CXL, when an eyelid speculum is left in place. ${ }^{10-12}$ In addition, the subsequent treatment duration over which the cornea remains exposed could also potentially affect the final pachymetry.

Currently, ultrasound pachymetry is the most commonly used method for intraoperative pachymetry measurements during CXL. However, pachymetry measurements using this method are subject to limitations: Only single point measurements can be obtained and it is necessary to have contact to obtain the measurements.

Anterior segment optical coherence tomography (AS-OCT) is a noninvasive imaging modality that can be used to obtain high-resolution, cross-sectional images of the anterior structures of the eye. ${ }^{13,14}$ In comparison with ultrasound pachymetry, AS-OCT has the advantage of providing a more detailed anatomical image of the cornea during procedure with a high degree of intraobserver repeatability and interobserver reproducibility of the pachymetric measurements. ${ }^{15,16}$

Recently, there have been increasing reports of the use of intraoperative OCT for pachymetry measurements during CXL. ${ }^{17-19}$ Here, we evaluate the feasibility of intraoperative OCT for pachymetry measurements during CXL, and we compare the effect of two variations of the method (speculum-on versus speculum-off during imbibition with riboflavin-dexran solution) on pachymetric measurements during the CXL procedure with the epithelium.

\section{Material and methods}

The study was designed as an open prospective study. The local ethical committee approved the study protocol and the study adhered to the tenets of the Declaration of Helsinki.

The preoperative evaluation included a complete ophthalmologic examination including refraction, uncorrected and best corrected visual acuity (UCVA and BCVA), best contact lens corrected visual acuity, and corneal topography and pachymetry with Pentacam HR (Oculus, Wetzlar, Germany).

The patients included in this study had ages between 18 and 35 years and clinically, topographically, and/or pachymetrically documented progression of keratoconus with a thinnest pachymetry measurement $>400 \mu \mathrm{m}$. All patients underwent CXL using $0.1 \%$ riboflavin in $20 \%$ dextran T-500 solution and an accelerated protocol (10 min of irradiation with a UV light source intensity of $9 \mathrm{~mW} / \mathrm{cm}^{2}$ ) with epithelial removal.

Exclusion criteria included the presence of corneal scarring, active allergic eye disease, concomitant ocular infections, or severe ocular surface diseases such as dry eye syndrome.

Eleven eyes of 11 consecutive patients in group 1 and 14 eyes of 14 consecutive patients in group 2 were enrolled in the study based on the routine practices of two surgeons. After epithelial removal, in group 1, the eyelid speculum was left in place during riboflavin imbibitions, and in group 2, the speculum was removed up until the time of irradiation. All other surgical parameters remained the same for both groups.

\section{Surgical technique}

All procedures were performed in the operating room under sterile conditions. The preoperative preparation consisted of the application of povidone-iodine (Betadine) and topical anesthesia with tetracaine $0.5 \%$ (SinaDaru, Iran) eye drops. After the insertion of the eyelid speculum, the epithelium was loosened with $18 \%$ ethanol for $20 \mathrm{~s}$ and the central $7 \mathrm{~mm}$ of the corneal epithelium was mechanically debrided. After epithelial removal, the eyelid speculum was left in place in group 1 , while the speculum was removed in group 2. During the imbibition phase, $0.1 \%$ riboflavin in $20 \%$ dextran T-500 (SinaDaru) solution was instilled every three minutes for a total of $30 \mathrm{~min}$. The irradiation was initiated after stromal saturation with riboflavin was verified by observing the presence of a yellow flare in the aqueous humor. All eyes received $9 \mathrm{~min}$ of irradiation with an intensity of $9 \mathrm{~mW} / \mathrm{cm}^{2}$ from a distance of $5 \mathrm{~cm}$ using the LightLink CXL (LightMed, San Clemente, CA, USA).

At the end of the procedure, a bandage contact lens was placed on the cornea. The postoperative medication included preservative-free artificial tears, Levofloxacin eye drops until the removal of the bandage contact lens at day 7 and betamethasone $0.1 \%$ started four times daily and tapered over a 1 month period.

Two surgeons, AS and RG, performed the procedures in groups 1 and 2, respectively. A similar method was used in both groups with the exception of different maintenance or removal of the eyelid speculum during the imbibition with riboflavin-dextran solution,

\section{Pachymetry measurements}

For the pachymetry measurements, a spectral domain OCT (iVue 100-2; Optovue, Fremont, CA, USA) was used. 
Pachymetry was performed with the patient sitting in front of the OCT device in the operating room at each stage of the procedure including the baseline, after epithelial removal, after $30 \mathrm{~min}$ of imbibition with riboflavin, and after UV irradiation. Patients were asked to look at a central fixation target of the device. Images were captured after proper alignment of the scan by observing a vertical flair reflection at the corneal vertex. Prior to the pachymetry measurements, to avoid possible interference between the pre-corneal riboflavin reflectivity and the OCT measurements, the corneal surface was rinsed with balanced salt solution. ${ }^{7}$ After each measurement, careful attention was paid to ensure the sterility of the procedure.

The corneal thinnest pachymetry (CTP), displayed in the pachymetric map was used to compare pachymetry changes during the procedure at each step between treatment groups.

\section{Statistical analysis}

For pachymetry assessments, a minimum sample size of 11 was estimated for each group to obtain a power of 0.80 for a two-sided test $(\alpha=0.05, \sigma=35 \mu \mathrm{m}$ based on Soeters et $a l^{11}$ to detect a difference of $35 \mu \mathrm{m}$ ). Statistical analysis of the data was performed using SPSS software (SPSS Inc., Chicago, IL, USA). Standard descriptive statistics were employed to describe the quantitative variables. The Kolmogorov-Smirnov test was used to evaluate the normal distribution of the data. Repeated measure analysis of variance was used to evaluate and compare the trend of changes in different stages of the procedure within and between two groups. Chi-square test was used to compare the proportion of patients with pachymetry less than $400 \mu$ mafter riboflavin instillation and UV irradiation between groups. A $P$-value $<0.05$ was considered to be statistically significant.

\section{Results}

Baseline characteristics of patients are shown in Table 1. There was not statistically significant difference in the demographic and baseline data between groups.

\section{CTP before epithelial debridement}

There was no statistically significant difference between the two groups. The mean CTP was $469.8 \pm 26.5 \mu \mathrm{m}$ in speculum-on group and $463.2 \pm 25.1 \mu \mathrm{m}$ in speculum-off group at baseline $(P=0.531)$.
Table 1 Baseline characteristics of keratoconic patients undergoing collagen cross-linking

\begin{tabular}{lccc}
\hline & Speculum-on $(\mathrm{n}=11)$ & Speculum-off $(\mathrm{n}-14)$ & P-value \\
\hline Age (years) & $23 \pm 4$ & $22 \pm 3$ & 0.727 \\
SE (D) & $-3.00 \pm 1.10$ & $-2.60 \pm 1.20$ & 0.646 \\
Kmax(D) & $55.20 \pm 5.67$ & $56.62 \pm 5.43$ & 0.552 \\
MCT ( $\mu \mathrm{m})$ & $469.8 \pm 26.5$ & $463.2 \pm 25.1$ & 0.531 \\
\hline
\end{tabular}

Abbreviations: SE, spherical equivalent; Kmax, maximum keratometry.

\section{CTP after epithelial debridement}

The CTP decreased significantly in both groups (both $P<0.001)$. The mean CTP decreased by $32.2 \pm 7.0 \mu \mathrm{m}$ in the speculum-on group and by $25.21 \pm 10.54 \mu \mathrm{m}$ in the speculum-off group. The mean reduction in CTP was not statistically different between the two groups $(P=0.072)$.

\section{CTP after 30 min of riboflavin drop instillation}

There was a statistically significant reduction in the mean CTP in the speculum-on group. The mean CTP reduced from $437.6 \pm 28.5 \mu \mathrm{m}$ to $354.3 \pm 28.4 \mu \mathrm{m}$ in this group $(P<0.001)$. However, the thinning of the CTP was not significant in the speculum-off group $(P=0.223)$, which reduced from $438.0 \pm 27.1 \mu \mathrm{m}$ to $421.6 \pm 54.0 \mu \mathrm{m}$. There was more thinning in the speculum-on group compared with the speculum-off group $(P<0.001)$. The proportion of the eyes with a corneal pachymetry less than $400 \mu \mathrm{m}$ was $90.9 \%$ in the speculum-on and $35.7 \%$ in the speculum-off group $(P=0.012)$.

\section{CTP after UV-A irradiation}

Corneal thinnest point increased by $12.8 \pm 16.2(P=0.025)$ in the speculum-on group and decreased by $-34.7 \pm 46.1$ $(P=0.015)$ in the speculum-off group. The difference in the changes was statistically significant between groups $(P=0.002)$. The final mean MCT was $367.1 \pm 31.8$ in speculum-on group and $386.9 \pm 34.0 \mu \mathrm{m}$ in speculum-off group $(P=0.152)$. The proportion of the eyes with a corneal pachymetry less than $400 \mu \mathrm{m}$ was $81.8 \%$ in the speculum-on and $71.4 \%$ in the speculum-off group $(P=0.661)$.

The change in mean corneal thickness in the thinnest point at certain time points during collagen cross-linking is illustrated in Figure 1 and summarized in Table 2. The representative images of changes in each group is represented in Figures 2 and 3.

\section{Discussion}

Our study has demonstrated the feasibility of intraoperative OCT as an invaluable tool for pachymetric 
assessments during CXL. We observed a significant change and thinning in corneal pachymetry during the procedure in both groups. The presence or absence of the eyelid speculum had a major impact on the pattern and magnitude of changes; the largest changes occurred in the speculum-on group during the imbibition phase with isotonic riboflavin-dextran solution. On the other hand in the speculum-off group, the effect of the irradiation phase was more pronounced on the corneal pachymetry compared with the speculum-on group.

During the riboflavin instillation phase, we observed a mean decrease in the corneal thinnest point of $83 \mu \mathrm{m}$ $(23.5 \%)$ in the speculum-on group. In previous studies with ultrasound pachymetry, Kymionis et al ${ }^{8}$ reported a $75 \mu \mathrm{m}(20 \%)$ decrease in the corneal CTP during the riboflavin instillation time. Schmidinger et al ${ }^{9}$ and Soeters et $a l^{10}$ reported a mean decrease of $59 \mu \mathrm{m}$ and $62 \mu \mathrm{m}$ respectively, during this phase; while the speculum-off group exhibited respectively $1 \%$ and $11 \mu \mathrm{m}$ increase in corneal pachymetry at the same time.

Our study results are also in agreement with previous studies using OCT for pachymetry measurements during CXL. Mazzotta et al ${ }^{7}$ reported a reduction of $144.43 \mu \mathrm{m}$ $(32.1 \%)$ in CTP measurements when an eyelid speculum was left in place; with the majority of the stromal shrinkage occurring in the first $10 \mathrm{~min}$ of the riboflavin instillation, Chow et al ${ }^{11}$ in their study of six eyes of six patients observed a $126 \mu \mathrm{m}$ and $66 \mu \mathrm{m}$ decrease in the CPT measurements in patients with and without lid speculum, respectively.

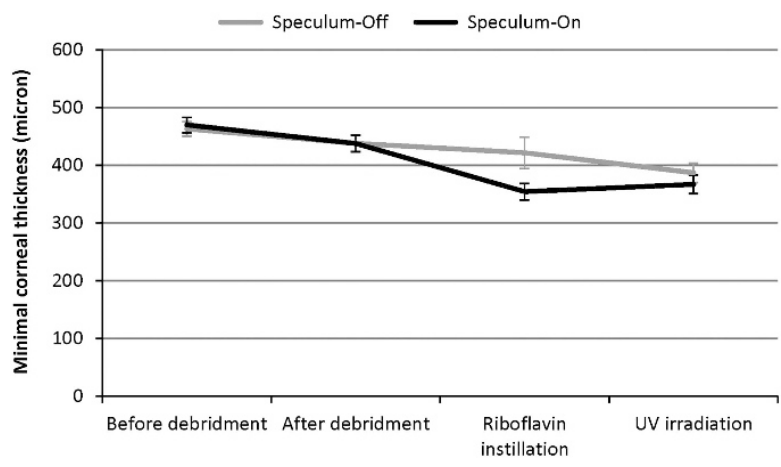

Figure 1 Mean thinnest corneal pachymetry measurements during CXL in the speculum-on and speculum-off groups during different stages of the procedure.
Despite the variations in the magnitude of the changes in previous reports that could be attributed to the baseline corneal thickness, the different riboflavin solutions available in the market, and measurement methods, these findings further highlight the role of corneal exposure in the imbibition phase as a critical factor influencing the corneal shrinkage observed intraoperatively during CXL with isotonic riboflavin-dextran solution and the epithelium-off protocol when an eyelid speculum is left in place. ${ }^{9,10,12}$

The corneal shrinkage during this period in the speculum-on group may be attributed to the osmotic properties of the isotonic riboflavin-dextran solution ${ }^{13}$ leading to corneal stromal thinning, in the context of the disruption of the corneal epithelial barrier and the presence of a normal endothelial pump function. ${ }^{14}$ In fact, the dextran in the standard isotonic riboflavin solution is not an essential component for the cross-linking effect, which explains the increasing reports of the use of other riboflavin solutions that are dextran-free or methylcellulose containing riboflavin solutions in recent reports. ${ }^{15-18}$

Another prominent finding was the observation of stronger influence of the irradiation phase on the corneal pachymetry in the speculum-off group (ie, $35 \mu \mathrm{m}$ decrease in corneal pachymetry. In contrast only a mild swelling of $13 \mu \mathrm{m}$ was noted in the speculum-on group. As a result of these changes, there was no significant difference in the final pachymetry between groups.

These findings are also in agreement with previous reports of a decrease in corneal pachymetry after reinsertion of the eyelid speculum in the irradiation phase $^{9,10}$ and minimal changes or mild swelling with the speculum-on method. 7,8

The observation of corneal thinning in the speculum-off group could be attributed to the effects of corneal exposure due to reinsertion of the eyelid speculum combined with the osmotic effects of riboflavin-dextran solution. In a recent study evaluating the intraoperative OCT pachymetry in patients undergoing dextran-free riboflavin accelerated CXL, Miguel Rechichi et al ${ }^{19}$ reported a stable pachymetry throughout the procedure, including the irradiation phase. So, it seems that the dextran in the isotonic riboflavin-dextran solution plays a

Table 2 Mean \pm SD change in minimal corneal thickness $(\mu \mathrm{m})$ at certain time points during accelerated corneal cross-linking

\begin{tabular}{lcrr}
\hline & Change in speculum-on group (percent) & Change in speculum-off group (percent) & P-value \\
\hline Before debridement & - & - & - \\
After debridement & $-32.2 \pm 7.0(7.3)$ & $-25.2 \pm 10.5(5.4)$ & 0.072 \\
After Riboflavin instillation & $-83.4 \pm 11.8(23.5)$ & $-16.4 \pm 48.1(3.9)$ & $<0.001$ \\
After UV-A irradiation & $+12.8 \pm 16.2(3.5)$ & $-34.7 \pm 46.1(9.0)$ & 0.002 \\
\hline
\end{tabular}



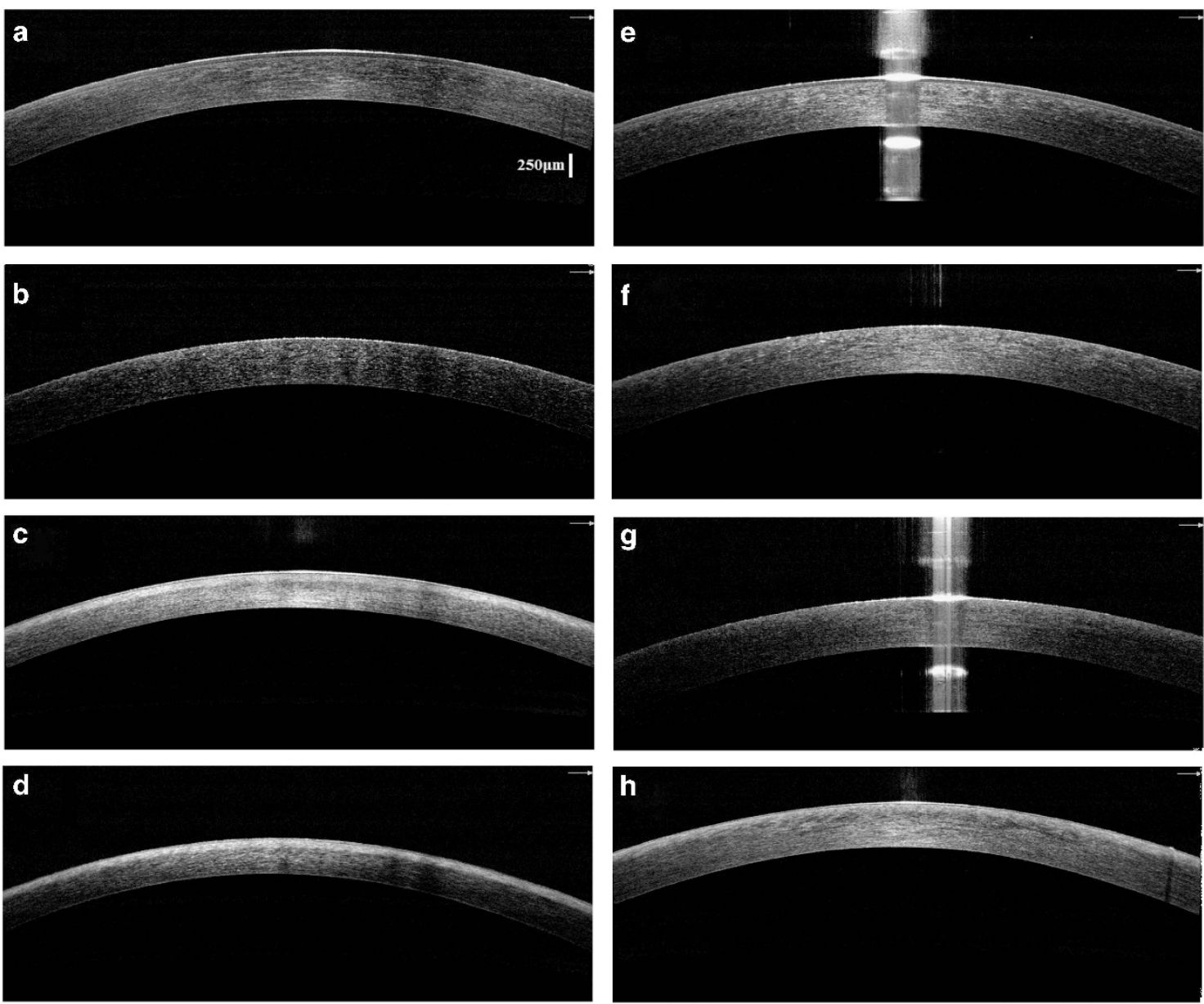

Figure 2 Sample OCT images corresponding to the baseline, after epithelial removal, after riboflavin-dextran instillation, and after UV irradiation two patients in the speculum-off $(\mathrm{a}-\mathrm{d})$ and speculum-on groups $(\mathrm{e}-\mathrm{h})$.

critical role in the corneal shrinkage during this period. In another study, Mazzotta et $a l^{7}$ reported that the most corneal shrinkage occurs in the first 10 min after insertion of the eyelid speculum. This is compatible with the results of our study where even an accelerated treatment (10 $\mathrm{min}$ ) was not able to maintain the full advantage of limiting corneal shrinkage in the riboflavin instillation period in the speculum-off group throughout the procedure.

On the other hand, the speculum-off group showed a mild increase in corneal pachymetry during the irradiation phase which may be attributed to the effects of CXL-induced corneal edema or a transient endothelial dysfunction. So, considering significant corneal shrinkage in the speculum-off group and a mild increase in corneal pachymetry in the speculum-on group, the final pachymetry was similar between groups.

The use of intraoperative OCT compared with the ultrasonic method for pachymetry measurements may be considered to be an advantage of our study.
Intraoperative OCT affords potential advantages such as higher-resolution imaging and the ability to visualize the corneal geometry and a high degree of reproducibility.

There are limitations to our study. Although our study possessed a sufficient sample size to detect the difference in changes during the riboflavin instillation phase, the observed trend of changes in some parameters such as the corneal thinning in the irradiation in the speculum-off method was not statistically significant, possibly because of small number of patients in this group. Studies with a larger number of cases will be useful for accurately characterizing these changes.

In conclusion, the results of our study demonstrate the potential feasibility and usefulness of the application of intraoperative OCT for pachymetric measurements during CXL. In general, the speculum-on group had a more stable corneal pachymetry during riboflavin instillation time. Considering that a minimum corneal thickness of $400 \mu \mathrm{m}$ is currently recommended for CXL to avoid the potential toxic effects of UV irradiation on the 
a

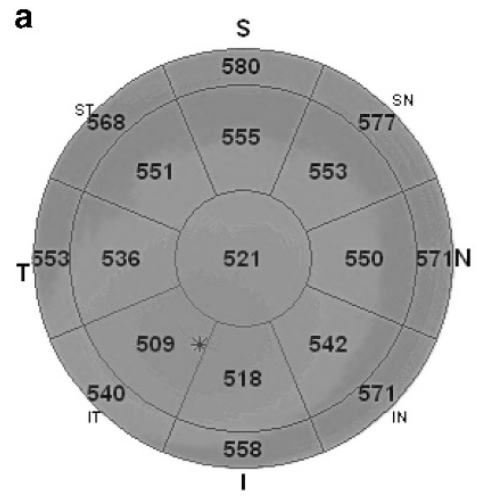

b

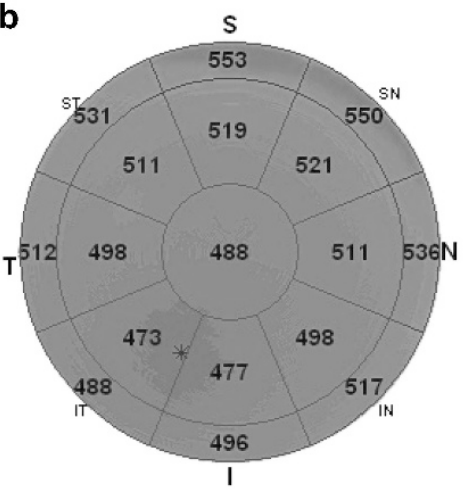

c

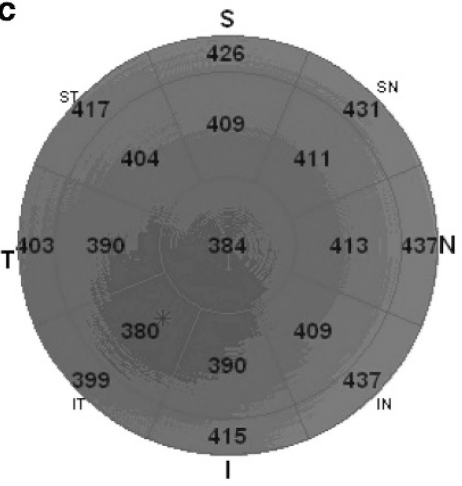

d

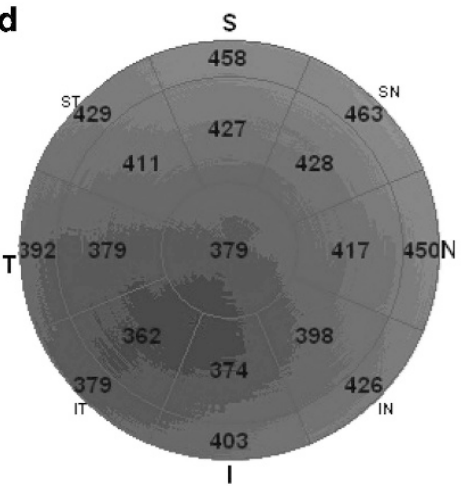

e

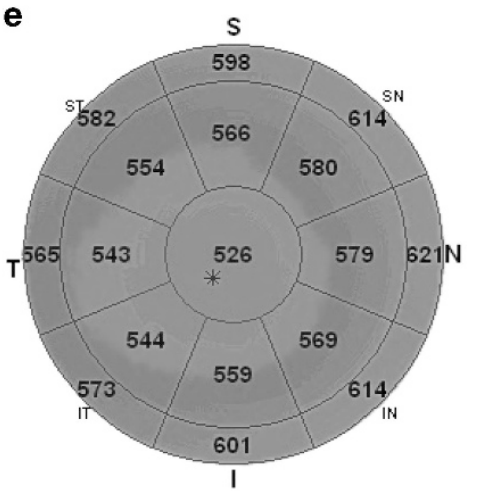

f

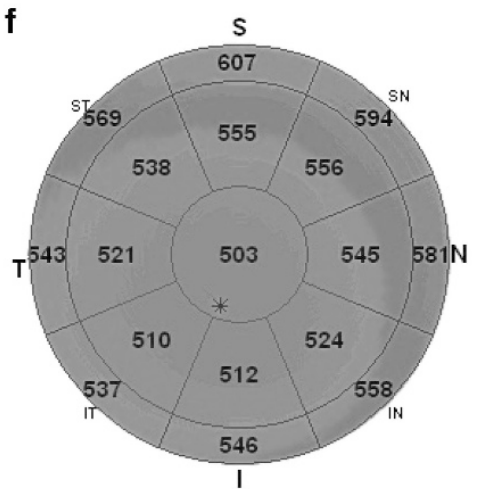

g

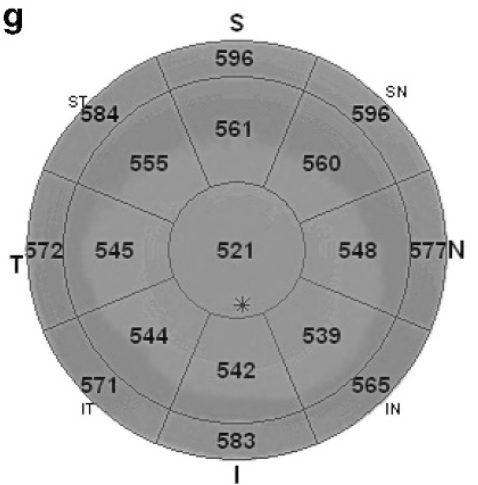

h

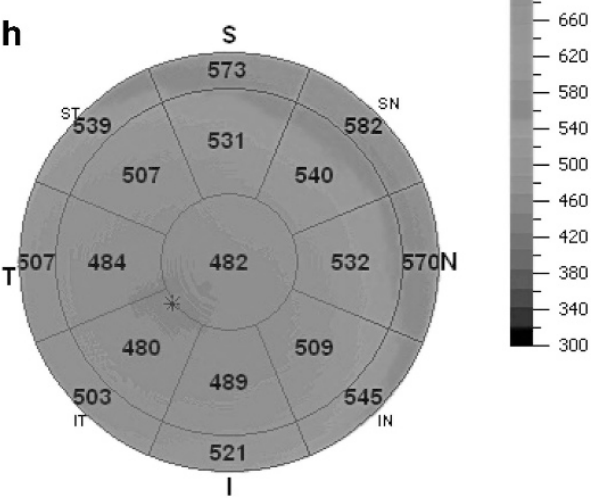

Figure 3 The corresponding pachymetry maps at the baseline, after epithelial removal, after riboflavin-dextran instillation, and after UV irradiation of the same patients in the speculum-off $(\mathrm{a}-\mathrm{d})$ and speculum-on $(\mathrm{e}-\mathrm{h})$ groups. Note the marked corneal thinning during riboflavin-dextran instillation in the speculum-on group. 
corneal endothelium, these findings further highlight the potential role of this strategy as a simple measure for limiting corneal shrinkage in this period and the need to convert to other modified methods such as the induction of corneal swelling by hypo-osmolar riboflavin solutions ${ }^{13}$ or contact lens-assisted CXL ${ }^{20}$ in these patients. However, the effect was not lasting for throughout the procedure and pronounced changes in corneal pachymetry were observed in both groups despite using a fast treatment protocol. These studies further highlight the limitations imposed by corneal stromal shrinkage using isotonic dextran containing riboflavin solution for CXL. Additional studies employing other riboflavin solutions and treatment protocols are warranted to further optimize the factors for a more stable corneal pachymetry during the CXL procedure.

\section{Summary}

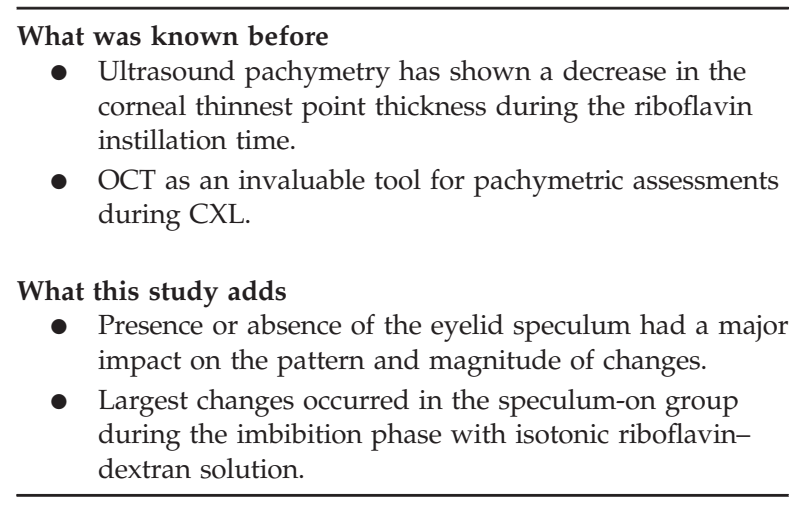

\section{Conflict of interest}

The authors declare no competing financial interest.

\section{References}

1 Rabinowitz YS. Keratoconus. Surv Ophthalmol 1998; 42(4): 297-319.

2 Wollensak G, Spoerl E, Seiler T. Riboflavin/ultraviolet-Ainduced collagen crosslinking for the treatment of keratoconus. Am J Ophthalmol 2003; 135(5): 620-627.

3 Wollensak G. Crosslinking treatment of progressive keratoconus: new hope. Curr Opin Ophthalmol 2006; 17(4): 356-360.

4 Theuring A, Spoerl E, Pillunat L, Raiskup F. [Corneal collagen cross-linking with riboflavin and ultraviolet-A light in progressive keratoconus: Results after 10-year follow-up]. Ophthalmologe 2015; 112(2): 140-147.

5 Hashemi H, Seyedian MA, Miraftab M, Fotouhi A, Asgari S. Corneal collagen cross-linking with riboflavin and ultraviolet A irradiation for keratoconus: long-term results. Ophthalmology 2013; 120(8): 1515-1520.
6 Spoerl E, Mrochen M, Sliney D, Trokel S, Seiler T. Safety of UVA-riboflavin cross-linking of the cornea. Cornea 2007; 26(4): 385-389.

7 Mazzotta C, Caragiuli S. Intraoperative corneal thickness measurement by optical coherence tomography in keratoconic patients undergoing corneal collagen cross-linking. Am J Ophthalmol 2014; 157(6): 1156-1162.

8 Kymionis GD, Kounis GA, Portaliou DM, Grentzelos MA, Karavitaki AE, Coskunseven E et al. Intraoperative pachymetric measurements during corneal collagen crosslinking with riboflavin and ultraviolet A irradiation. Ophthalmology 2009; 116(12): 2336-2339.

9 Schmidinger G, Pachala M, Prager F. Pachymetry changes during corneal crosslinking: effect of closed eyelids and hypotonic riboflavin solution. J Cataract Refract Surg 2013; 39(8): 1179-1183.

10 Soeters N, Van Bussel E, Van der Valk R, Van der Lelij A, Tahzib NG. Effect of the eyelid speculum on pachymetry during corneal collagen crosslinking in keratoconus patients. J Cataract Refract Surg 2014; 40(4): 575-581.

11 Chow VW, Biswas S, Yu M, Wong VW, Jhanji V. Intraoperative pachymetry using spectral-domain optical coherence tomography during accelerated corneal collagen crosslinking. Biomed Res Int 2013; 2013: 848363.

12 Li N, Peng X, Fan Z, Pang X, Xia Y. Variation in central corneal thickness during open-or closed-eye riboflavin instillation for corneal collagen cross-linking. Eye Sci 2013; 28(4): 185-189.

13 Hafezi F, Mrochen M, Iseli HP, Seiler T. Collagen crosslinking with ultraviolet-A and hypoosmolar riboflavin solution in thin corneas. J Cataract Refract Surg 2009; 35(4): 621-624.

14 Aurich H, Wirbelauer C, Jaroszewski J, Hartmann C, Pham DT. Continuous measurement of corneal dehydration with online optical coherence pachymetry. Cornea 2006; 25(2): 182-184.

15 Mazzotta C, Traversi C, Caragiuli S, Rechichi M. Pulsed vs continuous light accelerated corneal collagen crosslinking: in vivo qualitative investigation by confocal microscopy and corneal OCT. Eye 2014; 28(10): 1179-1183.

16 Mazzotta C, Baiocchi S, Caporossi T, Caragiuli S, Paradiso AL, Caporossi A. Riboflavin $0.1 \%(V i b e X)$ for the treatment of keratoconus. Expert Opin Orphan Drugs 2013; 1: 235-240.

17 Oltulu R, Satirtav G, Donbaloglu M, Kerimoglu H, Özkagnici A, Karaibrahimoglu A. Intraoperative corneal thickness monitoring during corneal collagen cross-linking with isotonic riboflavin solution with and without dextran. Cornea 2014; 33(11): 1164-1167.

18 Çınar Y, Cingü AK, Şahin A, Türkcü FM, Yüksel H, Caca I. Intraoperative corneal thickness measurements during corneal collagen cross-linking with isotonic riboflavin solution without dextran in corneal ectasia. Cutan Ocular Toxicol 2014; 33(1): 28-31.

19 Rechichi M, Mazzotta C, Daya S, Mencucci R, Lanza M, Meduri A. Intraoperative OCT. Pachymetry in patients undergoing dextran-free riboflavin UVA accelerated corneal collagen crosslinking. Curr Eye Res 2016; 41(10): 1310-1315.

20 Jacob S, Kumar DA, Agarwal A, Basu S, Sinha P, Agarwal A. Contact lens-assisted collagen cross-linking (CACXL): a new technique for cross-linking thin corneas. J Refract Surg 2014; 30(6): 366-372. 